\title{
Impact of watch and wait procedure after endoscopic detorsion for sigmoid volvulus in high risk dementia patients
}

\author{
Onur Ag, Yusuf Bozcan, Mürşit Dincer
}

Department of General Surgery, Firat University Hospital, Elazig, Turkey

Submitted: 19 November 2020

Accepted: 7 February 2021

Arch Med Sci Civil Dis 2021; 6: e12-e17

DOI: https://doi.org/10.5114/amscd.2021.105392

Copyright @ 2021 Termedia \& Banach

\section{Abstract}

Introduction: Volvulus is defined as an acute bowel dilatation, which must be treated after the diagnosis in a short time, which otherwise can cause necrosis and perforation of the bowel. Chronic constipation and neurological diseases are the most common causes in some countries. In this study, patients with sigmoid volvulus who were treated and followed up were examined, the association of it with neurological diseases was investigated, and the demographic characteristics of the patients, treatment approaches, and results were presented.

Material and methods: Patients who underwent colonic volvulus with dementia between January 2010 and August 2020 were retrospectively reviewed. The demographic characteristics and histopathological diagnosis of the patients were recorded. Differences in treatment modalities and results of patients with volvulus were statistically analyzed.

Results: Seventy-eight patients who had sigmoid volvulus and dementia were included the study. The median age was 82.0 years and males represented $71.8 \%$ of the patients. All the patients had abdominal pain, while the other common symptoms were vomiting, abdominal distension and constipation. The complication rate was statistically significantly higher in surgical treatment than endoscopic procedures and spontaneously detorsioned $(p=0.011)$. The median length of stay was statistically significantly different in the surgery group, endoscopic procedure group and spontaneously detorsioned group $(p<0.001)$.

Conclusions: The prevalence of volvulus is high in individuals with neurological disease. Successful results were obtained with non-surgical follow-up after endoscopic detorsion in the selected patient group in the results of this study, and mortality and morbidity were at lower rates than surgery.

Key words: dementia, volvulus, endoscopy.

\section{Introduction}

Volvulus occurs when the air-filled colonic segment torsions around the mesentery. It is defined as an acute bowel dilatation, which must be treated after the diagnosis in a short time, which otherwise can cause necrosis and perforation in the bowel. Volvulus causes $5-8 \%$ of all bowel obstructions with a male/female ratio of $1.4: 1$ [1]. A total of $90 \%$ of the cases are in the sigmoid colon, but it can also be seen in the cecum and transvers colon. Especially, mesenteric base stenosis and the presence of elongated sigmoid colon are the most common predisposing factors [2].

\author{
Corresponding author: \\ Mürşit Dincer \\ Department of \\ General Surgery \\ Firat University \\ Hospital \\ Elazig, Turkey \\ Phone: +905446422820 \\ E-mail: drmursitdincer@ \\ gmail.com
}


Also, the tendency to volvulus increases in chronic constipation and neurological diseases [3]. Chronic constipation and neurological diseases are the most common causes in some countries [1].

Constipation is one of the most common non-motor symptoms in Parkinson's disease, occurring in $50-80 \%$ of patients. It usually occurs in the early period of the disease, years before motor symptoms [4, 5]. Constipation may be accompanied by other gastrointestinal symptoms that are associated with intestinal motility. Pain, nausea, vomiting, distension, and total bowel obstruction affect $7 \%$ of Parkinson's disease patients. Anismus, abnormal contraction of the external anal sphincter, and problems in the puborectal muscles during defecation can be synergistic with constipation in $65 \%$ of Parkinson's disease patients. Although serious conditions such as megacolon, pseudo-obstruction, volvulus, and bowel perforation can occur, the exact incidence is not known [4]. In young patients, however, sigmoid volvulus is often associated with Hirschsprung's and Chagas disease [2].

Sigmoid volvulus often causes abdominal emergencies in the elderly, especially in individuals with psychiatric illness and dementia [6]. The findings of volvulus are the same as acute bowel obstruction. Patients are admitted with abdominal distension, nausea, and vomiting. Symptoms quickly progress towards generalized abdominal pain and tenderness. Fever and leukocytosis are the precursor of gangrene and perforation. Volvulus may spontaneously decrease; however, it often causes bowel obstruction, which can then cause strangulation, necrosis, and perforation. Physical examination, direct abdominal X-ray, and abdominal computed tomography $(\mathrm{CT})$ are useful in the diagnosis [3]. The first imaging method in volvulus is direct X-ray. The typical coffee-bean image makes the diagnosis easy. No further imaging methods are required to confirm the diagnosis in most cases [7]. After direct X-ray, CT establishes the diagnosis with approximately $100 \%$ sensitivity and $90 \%$ specificity $[8,9]$. However, colonoscopy is the most effective diagnostic method in the diagnosis of volvulus $[5,10]$.

Conservative methods such as endoscopic detorsion, fleet enema, and rectal tube application are used in the treatment of sigmoid volvulus. However, surgical methods such as resection and anastomosis, Hartman's procedure, and PEC may also be applied. Initial treatment is usually endoscopic decompression; but the risk of recurrence is high (40-90\%) [3]. Decompression can be performed with rigid proctoscopy, flexible sigmoidoscopy, and colonoscopy. The patient should be stabilized and an electric sigmoid colectomy should be performed after bowel preparation because of the high risk of recurrence. If there are clinical signs of necrosis and perforation, or strangulation in endoscopic imaging, emergency surgery should be scheduled without decompression. Hartman's procedure with end-colostomy is the safest surgical method for these patients. Large bowel resection is the gold standard in the treatment of recurrent sigmoid volvulus, but it was reported that the mortality rate in emergency resection increases up to $50 \%$ [3]. Percutaneous endoscopic colostomy (PEC) is considered as an alternative operation management in the elderly and in weak patients who are at high risk for surgery [3]. According to the data in recent series regarding the intervention with detorsion, the mortality is approximately $3 \%$ and mortality is $4 \%$. Hartman's procedure with end-colostomy is the safest surgical method in patients with emergency surgery scheduled for sigmoid volvulus. Percutaneous endoscopic colostomy provides alternative operation management for surgery in the elderly and in weak patients who are at very high risk [11-13]. In this study, patients with sigmoid volvulus who were treated and followed up were examined, the association of it with neurological diseases was investigated, and the demographic characteristics of the patients, treatment approaches, and results were presented.

\section{Material and methods}

Patients who underwent colonic volvulus with dementia between January 2010 and August 2020 were retrospectively reviewed. We assessed patients for eligibility in the study; after patients who had volvulus other than sigmoid colon were excluded from the study, 78 patients who had sigmoid volvulus and dementia were included in the study. The demographic characteristics and histopathological diagnosis of the patients were recorded. Differences in treatment modalities and results of patients with volvulus were statistically analyzed.

\section{Statistical analysis}

Statistical analyses were performed using SPSS version 23 (IBM Corp. in Armonk, NY). Kolmogorov-Smirnov and Shapiro-Wilk tests were used to evaluate the distribution of the data. Descriptive data are presented as frequencies $(n)$ and percentages (\%) for categorical variables, and median with interquartile range (IQR) for non-normally distributed numerical variables. Fisher's exact test was used for comparing categorical variables. Independent-samples Kruskal-Wallis test was used for comparing non-normally distributed data among treatment types, and Dunn's post-hoc test with Bonferroni correction was used in pairwise 
comparisons. $P<0.05$ was considered as statistically significant.

\section{Results}

This study was performed with dementia patients admitted to our general surgery department with colonic volvulus. The median age was 82.0 years and males comprised $71.8 \%$ of the patients. All the patients had abdominal pain, while the other common symptoms were vomiting, abdominal distension and constipation. The median time to symptom onset was 3.0 days. Eight patients had previous volvulus history and 23 had a prior intestinal operation. Of the patients, 15 had a concomitant/underlying neuropsychiatric disorder such as cerebrovascular disease, Parkinson, Alzheimer and cerebral palsy, and 19 had an underlying colonic disorder such as elongated sigmoid colon and colon cancer (Table I).

Of the patients, 56 underwent surgical procedures and 15 were treated with endoscopic procedures, while volvulus spontaneously detorsioned in 4 patients. Unfortunately, three patients could not be treated, and all those patients died. The

Table I. Demographics and baseline characteristics of the patients

\begin{tabular}{|c|c|}
\hline Variable & Value \\
\hline Age [years], median (IQR) & $82.0(58.5-87.0)$ \\
\hline \multicolumn{2}{|l|}{ Sex, $n(\%):$} \\
\hline Female & $22(28.2)$ \\
\hline Male & $56(71.8)$ \\
\hline \multicolumn{2}{|l|}{ Symptoms, $n(\%)$ : } \\
\hline Abdominal pain & $78(100.0)$ \\
\hline Vomiting & $56(71.8)$ \\
\hline Abdominal distension & $32(41.0)$ \\
\hline Obstipation & $14(17.9)$ \\
\hline Weight loss & $3(3.8)$ \\
\hline $\begin{array}{l}\text { Time to onset of symptoms [days], } \\
\text { median (IQR) }\end{array}$ & $3.0(2.0-3.3)$ \\
\hline Previous volvulus history, $n(\%)$ & $8(10.3)$ \\
\hline Previous intestinal surgery, $n(\%)$ & $23(29.5)$ \\
\hline $\begin{array}{l}\text { Presence of neuropsychiatric } \\
\text { disorder, } n(\%)\end{array}$ & $15(19.2)$ \\
\hline Presence of colonic disorder, $n(\%)$ & $19(24.4)$ \\
\hline
\end{tabular}

most prevalent complication related to treatment was respiratory distress, while the other common complications were poor general condition, anastomosis leak and ileus (Table II).

The complication rate was statistically significantly higher in surgical treatment than endoscopic procedures and spontaneously detorsioned $(p=0.011)$. However, recurrence rates were not statistically significantly different among the three treatment types (Table III).

The median length of stay was 10 days in the surgical patient group, 5 days in the endoscopic procedure group and one day in the spontaneously detorsioned group. This difference was statistically significant $(p<0.001)$. In the pairwise comparison, the length of stay was statistically significantly higher in the surgical procedure group than the endoscopic procedure group $(p=0.001)$ and spontaneously detorsioned group $(p=0.001)$. However, the length of stay did not differ significantly between the endoscopic procedure group and spontaneously detorsioned group (Table IV, Figure 1).

Overall in-hospital mortality was $24.4 \%$ and all the patients who could not be treated died in hospital. However, surgical procedures were more often fatal than endoscopic interventions. All the patients with volvulus that detorsioned spontaneously were discharged from the hospital (Table V).

Table II. Treatment types and complication rates

\begin{tabular}{|lc|}
\hline Variable & Value \\
\hline Treatment type, $n$ (\%): & $56(71.9)$ \\
\hline Surgical procedure & $15(19.2)$ \\
\hline Endoscopic procedure & $4(5.1)$ \\
\hline Detorsed spontaneously & $3(3.8)$ \\
\hline Unable to treat & $17(21.8)$ \\
\hline Complication, $n$ (\%): & $5(6.4)$ \\
\hline Respiratory distress & $4(5.1)$ \\
\hline Poor general condition & $3(3.8)$ \\
\hline Anastomosis leak & $2(2.6)$ \\
\hline Ileus & $2(2.6)$ \\
\hline Rectal bleeding & $1(1.3)$ \\
\hline Acute renal failure & $1(1.3)$ \\
\hline Anemia & \\
\hline Hypotension & \\
\hline
\end{tabular}

Table III. Comparison of complication rate and recurrence rate among the treatment types

\begin{tabular}{|lcccc|}
\hline Variable & $\begin{array}{c}\text { Surgical procedure } \\
(n=56)\end{array}$ & $\begin{array}{c}\text { Endoscopic } \\
\text { procedure } \\
(n=15)\end{array}$ & $\begin{array}{c}\text { Detorsed } \\
\text { spontaneously } \\
(n=4)\end{array}$ & $P_{\text {-value* }}$ \\
\hline Presence of complication, $n(\%)$ & $27(48.2)$ & $2(13.3)$ & $0(0.0)$ & 0.011 \\
\hline Recurrence, $n(\%)$ & $0(0.0)$ & $1(6.7)$ & $0(0.0)$ & 0.253 \\
\hline
\end{tabular}

${ }^{\star}$ Fisher's exact test was used. 
Table IV. Comparisons of median length of stay in hospital among treatment types

\begin{tabular}{|lcc|}
\hline Variable & Length of stay [day], median (IQR) & $P$-value \\
\hline \begin{tabular}{l} 
Treatment type: \\
\cline { 2 - 3 } Surgical procedure
\end{tabular} & $10.0(7.0-17.8)$ & \\
\hline Endoscopic procedure & $5.0(3.0-6.0)$ & \\
\hline Detorsed spontaneously & $1.0(1.0-1.8)$ & \\
\hline Pairwise comparisons: & & \\
\hline Surgical procedure-Endoscopic procedure & & \\
\hline Surgical procedure-Detorsed spontaneously & & \\
\hline Endoscopic procedure-Detorsed spontaneously & & \\
\end{tabular}

*Independent-samples Kruskal-Wallis test was used. ${ }^{* *}$ Dunn's pairwise post-hoc test with Bonferroni correction was used.

\section{Discussion}

Sigmoid volvulus accounts for an average of $5 \%$ of all bowel obstructions [10]. It is more common in men than in women. Similar to the literature, a total of $73 \%$ of the patients were male. According to previous studies, anatomical predisposing factors were found to be associated with a diet rich in fibrous foods, chronic constipation, past abdominal surgery, neurological or psychiatric diseases, high altitude, long-lasting bed rest, and mega-colon volvulus development. Chronic constipation and neurological diseases are common causes of it in the Western world [1]. In this study, there was a history of neurological diagnosis in $16(20 \%)$ patients, elongated sigmoid colon in 7 (8\%) patients, and abdominal surgery in 25 (35\%) patients.

The clinical symptoms and findings of volvulus are the same as other pathologies that progress with acute bowel obstruction, and are non-specific. Most patients present with abdominal pain, abdominal distension, nausea, and vomiting. In this study, symptoms consisted of similar complaints.

The patients were previously reported to present to a healthcare center with abdominal distension attacks in $30-41 \%$ of cases [14]. Symptoms can progress rapidly towards generalized abdominal pain and tenderness. In this rapidly evolving manifestation, the prognosis is poor. In this study, all of the patients underwent direct X-ray, 31 (38\%) patients were diagnosed with direct X-ray, 45 (56\%) patients were diagnosed with CT, and 4 (5\%) patients were diagnosed with endoscopic procedures. Colonoscopy can be considered as the first choice in cases of non-complicated sigmoid volvulus because it is both a diagnostic and

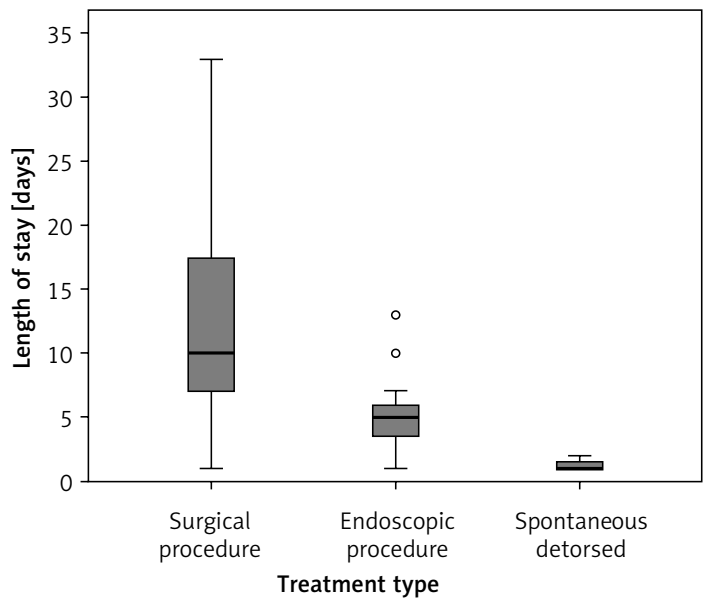

Figure 1. Comparisons of length of stay in hospital among treatment types

treatment tool. Colonoscopy examination not only evaluates the vitality of the sigmoid colon, but can also provide the detorsion of the volvulus. Endoscopic detorsion is a relatively easy, cost-effective, and minimally invasive procedure compared to other treatment methods, and has a success rate of $70-95 \%$.

Endoscopic detorsion was performed before the surgery in all patients in this study. A total of 9 (11\%) patients had recto-sigmoidoscopy, 11 (13\%) patients had detorsion with colonoscopy, and other treatment procedures were not used. In this study, 17 patients had end-side anastomosis, 12 patients had side-side anastomosis, 10 patients had end-end anastomosis, and 15 patients underwent Hartman's procedure. The PEC procedure was not performed on any patient. When the surgical treatment in emergency conditions and surgical procedures performed in elective

Table V. Comparison of in-hospital fatality rate of the patients

\begin{tabular}{|c|c|c|c|c|c|c|}
\hline Variable & $\begin{array}{c}\text { Surgical } \\
\text { procedure } \\
(n=56)\end{array}$ & $\begin{array}{l}\text { Endoscopic } \\
\text { procedure } \\
(n=15)\end{array}$ & $\begin{array}{c}\text { Detorsed } \\
\text { spontaneously } \\
(n=4)\end{array}$ & $\begin{array}{c}\text { Unable } \\
\text { to treat } \\
(n=3)\end{array}$ & $\begin{array}{c}\text { Total } \\
(n=78)\end{array}$ & $P$-value ${ }^{*}$ \\
\hline In-hospital fatality, $n(\%)$ & $14(25.0)$ & $2(13.3)$ & $0(0.0)$ & $3(100.0)$ & $19(24.4)$ & 0.020 \\
\hline
\end{tabular}


conditions after colonoscopy decompression are compared, it is found that there is significant differentiation of morbidity and mortality $[7,9,15$, $16]$. In this study, mortality after elective surgery was $10 \%(2 / 19)$, and mortality after emergency surgery was 33\% (10/35). Endoscopic decompression, which is the most effective non-surgical treatment method, is the first method that should be tried if there are no signs of perforation and necrosis in the initial evaluation of the patient [11]. However, it is observed in many studies that the chance of success varies between $20 \%$ and $70 \%$ in a wide range of colonoscopy detorsion methods $[8,13,15,17]$. For this reason, it is recommended to perform sigmoid colon resection in elective conditions after endoscopic detorsion [15]. Endoscopic detorsion was performed as the first intervention in all patients in this study, and success was achieved in 20 (25\%) patients. Endoscopic detorsion is a relatively safe procedure that has a mortality rate between $1 \%$ and $3 \%[12,17]$. Surgery is the first treatment option in recurring volvulus, because endoscopy is accompanied by a mortality rate as high as $20 \%$ [11]. In this study, surgical intervention was performed in 7 (77\%) of $9(11 \%)$ patients who had a previous history of volvulus, endoscopic intervention was performed in 2 (2.5\%) patients, 1 of the 2 patients who underwent detorsion died, 7 patients who underwent surgery had no mortality, and the mortality rate was $50 \%$. The mortality rate as a result of endoscopic interventions was $25 \%$ in this study (5 patients).

Endoscopic detorsion can be performed with rigid sigmoidoscopy as well as colonoscopy. However, flexible endoscopy is recommended rather than rigid endoscopy because of its low perforation rate, superior diagnostic performance and especially its superiority in assessing ischemia [13]. Rigid sigmoidoscopy may be insufficient in diagnosing the sigmoid volvulus, and especially ischemia in up to $24 \%$ of all cases. After endoscopic decompression, a rectal tube can be used for a period between 36 and $72 \mathrm{~h}$. It prevents re-torsion of the colon as well as providing rectal tube decompression. Detortion alone has a very high recurrence rate, as high as $74 \%(11,17)$. In the present study, recurrence was observed in 9 (11\%) patients.

Semi-elective sigmoidectomy can be performed after detorsion in terms of the etiology. However, such patients should have an elective sigmoid colectomy after stabilizing and after preparing the bowel because of the high risk of recurrence. In clinical terms, if there are signs of gangrene and perforation, and strangulation in endoscopic imaging, it is important to plan emergency surgery without decompression $[11,12,18]$. In this study, emergency surgery was scheduled for 35 (43\%) patients, and elective surgery was scheduled for 19 patients [19]. In the literature, total colonoscopy before surgery is recommended only if there are clinical or radiological suspicions regarding the underlying neoplasia [20, 21].

The postoperative mortality rate because of volvulus varies between $0 \%$ and $50 \%$, and was reported as $42 \%$ in necrotized patients [19, 21-24]. In this study, the mortality rate after the resection was $20 \%$. In the findings of exploration, 1 patient with bowel necrosis died. The retrospective design of the study and the small sample size were the negative aspects of this study.

In conclusion, the prevalence of volvulus is high in individuals with advanced age and neurological disease. Successful results were obtained with non-surgical follow-up after endoscopic detorsion in the selected patient group in the results of this study, and mortality and morbidity were at lower rates than surgery. However, randomized and controlled trials are needed for treatment selection to confirm the results of this study.

\section{Conflict of interest}

The authors declare no conflict of interest.

\section{References}

1. Ifversen AKW, Kjaer DW. More patients should undergo surgery after sigmoidvolvulus. World J Gastroenterol 2014; 20: 18384-9.

2. Perrot I, Fohlen A. Management of colonic volvulus in 2016. J Visc Surg 2016; 153: 183-92.

3. Frank L, Moran A. Use of percutaneous endoscopic colostomy (PEC) to treat sigmoid volvulus: a systematic review. Endosc Int Open 2016; 4: e737-41.

4. Poirier AA, Aubé B, Côté M, et al. Gastrointestinal dysfunctions in Parkinson's disease: symptoms and treatments. Parkinsons Dis 2016; 2016: 6762528.

5. Toebosch S, Tudyka V. Treatment of recurrent sigmoid volvulus in Parkinson's disease by percutaneous endoscopic colostomy. World I Gastroenterol 2012; 18: 5812-5.

6. Safioleas M, Chatziconstantinou C. Clinical considerations and therapeutic strategy for sigmoid volvulus in the elderly: a study of 33 cases. World J Gastroenterol 2007; 13: 921-4.

7. Renzulli P, Maurer CA, Netzer P, Büchler MW. Preoperative colonoscopic derotation is beneficial in acute colonic volvulus. Dig Surg 2002; 19: 223-9.

8. Shepherd JJ. Treatment of volvulus of sigmoid colon: a review of 425 cases. Br Med J 1968; 1: 280-3.

9. Shepherd JJ. The epidemiology and clinical presentation of sigmoid volvulus. Br J Surg 1969; 56: 353-9.

10. Hasnaoui H, Laytimi F, Elfellah Y, et al. Transverse colon volvulus presenting as bowel obstruction: a case report. J Med Case Rep 2019; 13: 156.

11. Grossmann EM, Longo WE, Stratton MD, Virgo KS, Johnson FE. Sigmoid volvulus in department of veterans affairs medical centers. Dis Colon Rectum 2000; 43: 414-8.

12. Oren D, Atamanalp SS, Aydinli B, et al. An algorithm for the management of sigmoid colon volvulus and the 
safety of primary resection: experience with 827 cases. Dis Colon Rectum 2007; 50: 489-97.

13. Turan $M$, Sen $M$, Karadayi A, et al. Our sigmoid colon volvulus experience and benefits of colonoscope in detortion process. Rev Esp Enferm Dig 2004; 96: 32-5.

14. Gingold D, Murrell Z. Management of colonic volvulus. Clin Colon Rectal Surg 2012; 25: 236-44.

15. Atamanalp SS. Treatment of sigmoid Volvulus: a singlecenter experience of 952 patients over 46.5 years. Tech Coloproctol 2013; 17: 561-9.

16. Atamanalp SS, Ozturk G. Sigmoid volvulus in the elderly: outcomes of a 43-year, 454-patient experience. Surg Today 2011; 41: 514-9.

17. Sutcliffe MM. Volvulus of the sigmoid colon. Br J Surg 1968; 55: 903-10.

18. Sule AZ, Iya D, Obekpa PO, Ogbonna B, Momoh JT, Ugwu BT. One-stage procedure in the management of acute sigmoid volvulus. J R Coll Surg Edinb 1999; 44: 164-6.

19. Ozdemir S, Aslar AK, Kuzu MA. Sigmoid volvulus: longterm surgical outcomes and review of the literature. S Afr J Surg 2012; 50: 9-15.

20. Larkin JO, Thekiso TB, Waldron R, Barry K, Eustace PW. Recurrent sigmoid volvulus-early resection may obviate later emergency surgery and reduce morbidity and mortality. Ann R Coll Surg Engl 2009; 91: 205-9.

21. Swenson BR, Kwaan MR, Burkart NE, et al. Colonic volvulus: presentation and management in metropolitan Minnesota, United States. Dis Colon Rectum 2012; 55: 444-9.

22. Halabi WJ, Jafari MD, Kang CY, et al. Colonic volvulus in the United States: trends, outcomes, and predictors of mortality. Ann Surg 2014; 259: 293-301.

23. Dolejs SC, Guzman MJ, Fajardo AD, Holcomb BK, Robb BW, Waters JA. Contemporary management of sigmoid volvulus. J Gastrointest Surg 2018; 22: 1404-11.

24. Bhatnagar BNS, Sharma CLN, Gautam A, Kakar A, Reddy DCS. Gangrenous sigmoid volvulus: a clinical study of 76 patients. Int J Colorectal Dis 2014; 19: 134-42. 